\title{
WETSEMTM - A New Technology for High Resolution Imaging of Fully Hydrated Biological
}

\section{Samples}

A. Nechushtan, A. Vainshtein, M. Horowitz, A. Sabban, O. Zik.

Electron microscopy is the prime tool for the study of biological ultra structures. Biological specimens are hydrated in their natural state. They therefore cannot be placed in the vacuumed chamber of a conventional Scanning Electron microscope (SEM), since samples will be dried, water would evaporate and interfere with the specimen-emitted electrons and the detectors. Moreover, its routine use in cell biology and histology is hampered by lengthy and potentially destructive sample preparation procedures especially de-hydration of samples.

We report here the QX-capsule, based on WETSEM ${ }^{\mathrm{TM}}$, a new technology that allows direct imaging of fully hydrated biological samples in a SEM. This new imaging technology offers unique advantages:

1. Imaging is preformed at atmospheric pressure with wide variety of the user's preferred temperature.

2. Sample preparation involves only liquid handling, obviating the need for drying, embedding, sectioning or coating. This makes the wet EM easily accessible for routine and reproducible imaging with deformations and other artifacts.

3. Unstained or live samples could be imaged if sufficient contrast could be obtained.

Enhancement of sample contrast with a wide variety of EM stains is straightforward.

4. The limited penetration of electrons into the sample allows visualization of wet, unembedded tissues without thin sectioning: only the outer layer of a few micrometers is visualized. 
5. The QX-capsule serves as a miniature cell culture dish, which allows similar handling and experimental manipulations as being done with regular cell cultures. These include light/fluorescence microscope use, centrifugation, transfection, differentiation, immunolabeling and staining. Images of cultured cells, tissue biopsies as well as microorganism reveal details of subcellular organelles, molecular localization, extracellular matrix, and tissue architecture. These demonstrate the utility of the technique for a wide range of applications. 